\title{
Interesting consequences of brane cosmology
}

\author{
Anupam Mazumdar \\ Astrophysics Group, Blackett Laboratory, Imperial College, London SW7 2BZ, United Kingdom
}

(Received 26 February 2001; published 27 June 2001)

\begin{abstract}
We discuss cosmology in four dimensions within the context of a brane-world scenario. Such models can predict chaotic inflation with very low reheat temperature depending on the brane tension. We notice that the gravitino abundance is different in brane-world cosmology and by tuning the brane tension it is possible to get an extremely low abundance. We also study Affleck-Dine baryogenesis in our toy model.
\end{abstract}

DOI: $10.1103 /$ PhysRevD.64.027304

PACS number(s): $98.80 . \mathrm{Cq}, 11.10 . \mathrm{Kk}$

Recently there has been renewed interest in perceiving the four-dimensional world which is in the form of a threedimensional hypersurface along with time embedded in a higher-dimensional space-time. Such a claim has a pedigree from the strongly coupled sector of $E_{8} \times E_{8}$ hetrotic string theory which can be described by a field theory living in an 11-dimensional space-time [1]. The 11-dimensional world is comprised of two 10-dimensional hypersurfaces embedded on an orbifold fixed point, where fields are assumed to be confined to the hypersurfaces which are known to be 9 branes in this scenario. After compactifying the 11dimensional theory on a Calabi-Yau threefold, one obtains an effective 5-dimensional theory [2], which has the structure of two 3 branes situated on the orbifold boundaries. The theory allows $N=1$ supergravity with gauge and chiral multiplets on the two 3 branes. Thus, it is possible to get phenomenologically interesting $N=1$ supergravity in four dimensions from hetrotic string theory. The low energy theory in four dimensions also allows a number of cosmological implications and in the recent past some attempts have been made to understand the cosmology [3].

In this paper we consider a very simple toy model in 5 dimensions and we assume that we reside in one of the two 3 branes which are separated by a distance. In this setup it has been realized that the effective 4-dimensional cosmology is nonconventional [4]. The Friedmann equation is modified due to localization of the fields on the brane and also due to presence of the second brane. The extra 5th dimension is assumed to have orbifold symmetry $y=-y$, and static in our case. The main goal of this paper is to point out some of the interesting implications of the brane cosmology taking place at energy scales below four-dimensional Planck mass and above the nucleosynthesis scale. It is fairly well recognized that the root of most of the nagging problems of the presently observed universe have some relation to the early universe. We mention two of them here. The present universe seems to be extremely flat, isotropic, and homogeneous. A small inhomogeneity is measured to be one part in $10^{5}$ by the Cosmic Explorer Background (COBE) satellite, and the second startling observation is that the present observable universe has a small baryon asymmetry which is noted to be roughly one part in $10^{10}$, measured from the abundances of light elements synthesized at the time of nucleosynthesis. A small inhomogeneity of the Universe can be explained by quantum fluctuations of the scalar fields during inflation. While the observed baryon asymmetry can also be explained quite el- egantly in the early universe because of the presence of a preferred time and the expansion of the Universe which leads to out-of-equilibrium decay of massive particles via explicit $C P$ violation interactions. In this paper we will consider one such example of baryogenesis in supersymmetric theories which is known as the Affleck-Dine (AD) mechanism [5]. Strictly speaking we will be treating the branes as hypersurfaces. We will be assuming that initial configuration of the branes are supersymmetric and due to some known or unknown reasons, supersymmetry is broken at a suitable scale to solve the hierarchy problem in the Planck brane where we reside. Regarding this we are assuming that our setup has two branes with opposite brane tensions and a negative bulk cosmological constant. In this respect our discussion could as well be generalizable to the configuration where gravity can be localized on the Planck brane [6] and some attempts have been made to supersymmetrize the two branes [7]. However, some formal aspects of supersymmetrizing infinitely thin branes are still under extensive study (see Refs. [8,9] and, more recently, Ref. [10]).

A simple isotropic and homogeneous cosmology can be described by the expansion parameter known as the Hubble parameter. It has been noticed that the two branes with opposite brane tensions can cancel the negative bulk cosmological constant [11] to give rise to a simple modification to the expansion equation. The Friedmann equation in the Planck brane is given by

$$
H^{2}=\frac{8 \pi}{3 M_{p}^{2}} \rho\left[1+\frac{\rho}{2 \lambda}\right],
$$

where $\rho$ is the energy density of the matter stuck to the brane. The brane tension $\lambda$ relates the four-dimensional Planck mass $M_{p} \approx 10^{19} \mathrm{GeV}$ to the five-dimensional Planck scale $M_{5}$ via

$$
M_{p}=\sqrt{\frac{3}{4 \pi}}\left(\frac{M_{5}^{2}}{\sqrt{\lambda}}\right) M_{5} .
$$

It is noticeable from Eq. (1) that there is an extra contribution to the right-hand side of the Friedmann equation. If we demand that successful nucleosynthesis occurs then the second term proportional to $\rho^{2}$ has to play a negligible role at a scale $\sim \mathcal{O}(\mathrm{MeV})$, corresponding to the era of big bang nucleosynthesis. Thus we have to assume that the modified 
Friedmann equation paves the usual term in the right-hand side of Eq. (1), which is just linear in energy density. This naturally leads to constraining the brane tension as $\lambda$ $>(1 \mathrm{MeV})^{4}$. This naturally leads to constraining the brane tension as $\lambda>(1 \mathrm{MeV})^{4}$; for better bounds see Ref. [12]. This means that the Universe evolves exactly in a familiar fashion even in the presence of branes at energy scales lower than a $\mathrm{MeV}$. However, there could be a significant departure from the usual lore at very high energies, especially when $2 \lambda$ $<\rho$. In this regime the expansion rate of the Universe is certainly dominated by the $\rho^{2}$ term in the right-hand side of Eq. (1). Our aim is to illustrate that perhaps we can accommodate the nonconventional term in Eq. (1) for solving some of the problems, such as excess gravitino production during reheating.

The energy conservation equation for the matter which is strictly residing within our brane is given by $\dot{\rho}+3 H(\rho+p)$ $=0$. This has an obvious consequence for the scalar field dominating the early universe during the inflationary phase. It has been pointed out in Refs. $[4,13]$ that inflation is well supported by $\rho^{2}$ contribution because of the dominance of the friction term leads to many $e$-foldings of inflation. For our purpose it is the last 50-60e-foldings of inflation should be sufficient enough to form structures in the Universe. The possibility of chaotic inflation with massive inflaton field $\left[V(\phi)=m^{2} \phi^{2} / 2\right]$ has been discussed in Ref. [13]. The density perturbation produced by the scalar field $\phi$ during inflation has been compared to that of the COBE result and it has been realized that chaotic inflation can occur for field values below the four-dimensional Planck mass $\phi_{\mathrm{COBE}}$ $\approx 10^{2} M_{p}^{1 / 3} \lambda^{1 / 6}<M_{p}$, but above the five dimensional scale $M_{5}$. The mass of the inflaton field has also been found to be constrained $m \approx 5 \times 10^{-5} M_{5}$, which essentially translates to $m \approx 10^{-5} M_{p}^{1 / 3} \lambda^{1 / 6}$ from Eq. (2). Hence, for $\lambda \approx \mathcal{O}(\mathrm{GeV})^{4}$, the mass could be $m \sim \mathcal{O}(10) \mathrm{GeV}$, and $\phi_{\mathrm{COBE}}$ $\sim \mathcal{O}\left(10^{8}\right) \mathrm{GeV}$. Thus the scale of inflation is determined by the brane tension and depending on its value inflation could take place at extremely low scale. One of the most important consequence of having inflation at a low scale is the low reheat temperature and various other physical implications which we will describe next.

It is known to us that inflation leads to an extremely cold universe because the entropy generated before and during inflation redshifts away, thus it is necessary to attain thermalization at a scale above the nucleosynthesis scale to preserve the successes of the big bang model. We notice, after the end of inflation the scalar field begins oscillating coherently at the bottom of the potential, and for the massive inflaton the average pressure density vanishes during the oscillations, thus leading $\rho_{\phi} \propto a^{-3}$, where $a$ is the scale factor. If we denote $\rho_{\phi i}$ and $a_{i}$ as the inflaton energy density and the scale factor at the beginning of the coherent oscillations, then the Hubble expansion is given by $H^{2}(a) \approx\left(8 \pi / 3 M_{p}^{2}\right)\left(\rho_{\phi i}^{2} /\right.$ $2 \lambda)\left(a_{i} / a\right)^{6}$. If the decay rate of the inflaton is denoted by $\Gamma_{\phi}$, then equating $H(a)$ to $\Gamma_{\phi}$ leads to an expression for the scale factors. If we assume reheating occurs with the energy density in radiation $\rho_{r}=\left(\pi^{2} / 30\right) g_{*} T_{\mathrm{rh}}^{4}$, where $g_{*}$ is the relativistic degrees of freedom then the reheat temperature $T_{\mathrm{rh}}$ is given by

$$
\begin{aligned}
T_{\mathrm{rh}} & \approx\left(\frac{\Gamma_{\phi} M_{p} \sqrt{\lambda}}{g_{*}}\right)^{1 / 4} \sim\left(10^{-5 / 4} M_{p}^{1 / 3} \lambda^{1 / 6}\right)\left(\frac{\alpha}{g_{*}}\right)^{1 / 4} \\
& \approx 10^{15 / 4} m_{\phi}\left(\frac{\alpha}{g_{*}}\right)^{1 / 4},
\end{aligned}
$$

where we have assumed massive boson decay $\Gamma_{\phi} \approx \alpha m_{\phi}$ with a Yukawa coupling $\alpha$, and, while deriving the last expression in Eq. (3), we have taken $m_{\phi} \approx 10^{-5} M_{p}^{1 / 3} \lambda^{1 / 6}$. We see that the reheat temperature is proportional to the mass of the inflaton. For the brane tension $\lambda \sim \mathcal{O}(1) \mathrm{GeV}$, reheat temperature could be $T_{\mathrm{rh}} \approx \mathcal{O}\left(10^{3}\right) \mathrm{GeV}$, assuming $g_{*}$ $\sim \mathcal{O}(100)$ and $\alpha \approx 0.01$. However, the reheat temperature is always more than the brane tension. This is a direct consequence of inflation occurring at low scales. Inflation at such a scale is desirable from the point of view of nucleosynthesis which we briefly describe here.

If we believe that supersymmetry is needed to solve the hierarchy between the electro-weak scale and the fourdimensional Planck mass then the gravitino mass must be no higher than $\sim 1 \mathrm{TeV}$. Since we know that gravitino coupling to matter is Planck mass suppressed, the life time of gravitino at rest is quite long $\tau_{3 / 2} \sim M_{p}^{2} / m_{3 / 2}^{3}$ $\sim 10^{5}\left(m_{3 / 2} / \mathrm{TeV}\right)^{-3} \mathrm{sec}[15]$. If the gravitino decays to either gauge bosons and its gaugino partner, or, if it decays to energetic photons, synthesis of light elements can be in danger by changing the number density of baryon to photon ratio required for a successful nucleosynthesis. However, if the Universe thermalizes at a temperature which is as low as $\mathcal{O}\left(10^{3}\right) \mathrm{GeV}$, the thermal production of gravitinos is also suppressed, but gravitinos could also be produced nonperturbatively during preheating [16], which we do not consider here. The thermal production of gravitino usually involves $2 \rightarrow 2$ processes involving gauge bosons and gauginos during reheating. In the brane-world scenario it is very likely that the bulk is also supersymmetric and in that case there is a possibility to excite the Kaluza-Klein gravitino modes. At the Planck brane these modes are coupled to the matter field with Planck mass suppressed interactions and it could be very interesting to analyze them separately, there has been some discussion upon localization of the zero mode gravitino in this context [17]. In this paper we do not study exciting the Kaluza-Klein gravitino modes, however, if the formal approach to study them becomes clear then it is worth investigating this issue separately, because they are likely to increase the gravitino abundance and thus likely to pose a bigger challenge to nucleosynthesis.

In order to study the gravitino abundance by strictly assuming that there is no gravitino contribution from the bulk, we need to study the Boltzmann equation for the gravitino number density $n_{3 / 2}$ in $3+1$ dimensions [14]:

$$
\frac{d n_{3 / 2}}{d t}+3 H n_{3 / 2}=\left\langle\Sigma_{\text {tot }} v_{\text {rel }}\right\rangle n_{\mathrm{rad}}^{2}-\frac{m_{3 / 2}}{\left\langle E_{3 / 2}\right\rangle} \frac{n_{3 / 2}}{\tau_{3 / 2}},
$$

where $\langle\cdots\rangle$ represents thermal average, $n_{\text {rad }}$ is the number density of relativistic particles $n_{\text {rad }} \propto T^{3}, v_{\text {rel }}$ is the relative velocity of the scattering radiation which in our case $\left\langle v_{\text {rel }}\right\rangle$ 
$=1$, and the factor $m_{3 / 2} /\left\langle E_{3 / 2}\right\rangle$ is the average Lorenz factor. We notice that in radiation era the nonconventional brane cosmology gives the following Hubble rate of expansion:

$$
H \approx\left(\frac{4 \pi^{5}}{3}\right)^{1 / 2} \frac{g_{*}}{30} \frac{T^{4}}{\sqrt{\lambda} M_{p}} .
$$

In supersymmetric version $g_{*} \sim 300$ provided the reheat temperature is more than the masses of the superpartners. It is worth mentioning that the scale factor during radiation era follows $a(t) \propto t^{1 / 4}$, which is contrary to the standard big bang scenario where $a(t) \propto t^{1 / 2}$. However, we must not forget that the derivation is based on the fact that we are in a regime where $\rho>2 \lambda$. In Eq. (4), after the end of inflation the first term in the right-hand side dominates the second. If we assume adiabatic expansion of the Universe $a \propto T^{-1}$, then we can rewrite Eq. (4) as $Y_{3 / 2}=\left(n_{3 / 2} / n_{\text {rad }}\right)$. We yield $d Y_{3 / 2} / d T$ $\approx-\left(\left\langle\Sigma_{\text {tot }}\right\rangle n_{\text {rad }} / H T\right)$. We notice that we can integrate the temperature dependence from this equation, and, we mention here that the above expression is exactly the same as in the standard big bang case [14]. However, this equation does not produce the correct value of $Y_{3 / 2}$, since the true conserved quantity is the entropy per comoving volume. In our case if we assume the gravitinos do not decay within the time frame we are interested in, then we may be able to get the abundance expression at two different temperatures:

$$
Y_{3 / 2}(T) \approx \frac{g_{*}(T)}{g_{*}\left(T_{\mathrm{rh}}\right)} \frac{n_{\mathrm{rad}}\left(T_{\mathrm{rh}}\right)\left\langle\Sigma_{\mathrm{tot}}\right\rangle}{H\left(T_{\mathrm{rh}}\right)} .
$$

Here we assume that the initial abundance of gravitinos at $T_{\mathrm{rh}}$ is known to us, and the dilution factor $g_{*}(T) / g_{*}\left(T_{\mathrm{rh}}\right)$ takes care of the decrease in the relativistic degrees of freedom. The total cross section $\Sigma_{\text {tot }} \propto 1 / M_{p}^{2}$, and $n_{\text {rad }}\left(T_{\text {rh }}\right) \propto T_{\text {rh }}^{3}$, we finally get an expression for the gravitino abundance at temperature $T$

$$
Y_{3 / 2}(T \ll 1 \mathrm{MeV}) \approx 10^{-3} \frac{\sqrt{\lambda}}{T_{\mathrm{rh}} M_{p}} .
$$

The above expression is an important one and now we are in a position to estimate the abundance for gravitinos. First of all we mention that the abundance equation is in stark contrast to the conventional one $Y_{3 / 2} \approx 10^{-2}\left(T_{\mathrm{rh}} / M_{p}\right)$, where the reheat temperature appears in the numerator rather than in denominator. If we assume that after their creation during reheating their number density is conserved, then for $T_{\text {rh }}$ $\approx 10^{3} \mathrm{GeV}$ and $\lambda \approx(1 \mathrm{GeV})^{4}$, we get an extremely small abundance of gravitinos $Y_{3 / 2} \approx 10^{-25}$. However, for similar reheat temperature, the conventional big bang cosmology would predict the abundance $Y_{3 / 2} \approx 10^{-18}$. Thus we find extremely low abundance of gravitinos in our case. However, we need to be cautious. The abundance depends on the brane tension and it can be evaluated easily, that increase in brane tension leads to increase in the mass of the inflaton and also the reheat temperature. This eventually leads to extremely high abundance of gravitinos during reheating compared to the ordinary big bang case. This could be a potent problem for intermediate range five-dimensional Planck mass, which is a common feature in M-theory compactifications. So, all is not well with the brane cosmology, however, for small brane tensions, $\rho^{2}$ contribution to the Friedmann equation could be beneficial. In order to be a successful candidate for small brane tensions, the issue of baryogenesis becomes very important and this is the discussion we follow next.

An important mechanism for generating baryon asymmetry is through the decay of sfermion condensate proposed in Ref. [5], known as the AD mechanism. Let us consider the sfermion condensate denoted by $\psi$ and a simple potential for $\psi$ which is lifted by breaking supersymmetry at a suitable scale $V \approx \tilde{m}^{2} \psi^{2}$, where $\tilde{m}$ is related to the supersymmetry breaking scale. A large baryon asymmetry can be generated if there is a baryon number violating operator, such as $\langle A\rangle$ $\neq 0$. The baryon number density stored in the sfermion oscillations is given by [5]

$$
n_{B}=\epsilon\left(\frac{\psi_{0}^{2}}{M_{G}^{2}}\right) \frac{\rho_{\psi}}{\tilde{m}}
$$

where $\psi_{0}$ is the initial amplitude of the sfermion oscillations, $M_{G}$ can be assumed to be an intermediate scale, this could be supersymmetric grand unification scale. $\epsilon\left(\psi_{0}^{2} / M_{G}^{2}\right)$ is the net baryon number generated by the decay of $\psi$. As we know in general the inflaton begins oscillating when $H \sim m_{\phi}$ at $a$ $=a_{\phi}$ and oscillations of the sfermion begin quite late when $H \sim \tilde{m}$ at $a=a_{\psi}$. One of the most important conditions to realize the $\mathrm{AD}$ baryogenesis is that the thermalization due to the decay products of the inflaton field must take place after the decay of the AD field, and, $\rho_{r \phi}>\rho_{\psi}$, where $\rho_{r \phi}$ is the energy density in radiation after the inflaton decay. This is mainly required to prevent washing out the baryon asymmetry. This tells us that the inflaton should decay very slowly and possibly via gravitational interactions, however, if this is so, then most probably the Universe would undergo transition from nonconventional to the standard one while the process of reheating. This will happen when $\rho_{\phi}$ $\approx m_{\phi}^{2} \phi^{2}\left(a_{\phi} / a\right)^{3} \sim \lambda$ at $a=a_{\lambda}=\left(m_{\phi}^{2} \phi^{2} / \lambda\right)^{1 / 3} a_{\phi}$. We picture a situation where the Universe began with a nonconventional cosmology, then after the end of inflation the inflaton begins oscillating, but the Universe is still nonconventional. When the Hubble parameter drops to a value $H \sim \tilde{m}$ the oscillations in the $\mathrm{AD}$ field begins and at this time also the Universe is nonconventional. However, soon after oscillations in the AD field is induced, the transition from nonconventional to the standard cosmology paves its way. Since the mass of the AD field is $\tilde{m} \sim m_{3 / 2}<m_{\phi}$ small compared to the mass of the inflaton, the oscillations in the AD field begin after the inflaton oscillations. This can be estimated by taking $H \sim \tilde{m}$. Since this happens when the Universe is nonconventional, $H \approx\left(m_{\phi}^{2} \phi^{2} / M_{p} \sqrt{\lambda}\right)\left(a_{\phi} / a\right)^{3} \sim \tilde{m}$. We can estimate the scale factor when this happens $a=a_{\psi}=\left(\sqrt{\lambda} / M_{p} \tilde{m}\right)^{1 / 3} a_{\lambda}$. It can be verified easily that $a_{\psi}<a_{\lambda}$. However, this restricts the fivedimensional Planck mass $M_{5}<10^{14} \mathrm{GeV}$. After $a_{\lambda}$ the cosmology becomes the standard one and the Hubble rate is given by $H \propto \sqrt{\rho} / M_{p}$. In our setup the inflaton decays when the Universe is already in the standard cosmology, thus we can estimate the scale factor when this happens by equating 
the Hubble parameter to the decay rate of the inflaton; $H$ $\approx\left(m_{\phi} \phi / M_{p}\right)\left(a_{\phi} / a_{\lambda}\right)^{3 / 2}\left(a_{\lambda} / a\right)^{3 / 2} \sim \Gamma_{\phi}=\left(m_{\phi}^{3} / M_{p}^{2}\right)$. Notice that the decay rate of the inflaton is via the gravitational coupling. This yields $a=a_{d \phi}=\left(\lambda M_{p}^{2} / m_{\phi}^{6}\right)^{1 / 3} a_{\lambda}$. It can be verified that $a_{\phi}<a_{\psi}<a_{\lambda}<a_{d \phi}$, this also requires to use the constraint on the mass of the inflaton; $m_{\phi} \sim 10^{-5} M_{p}^{1 / 3} \lambda^{1 / 6}$ [13]. During the oscillations of the AD field, the energy density decreases in the same fashion as in the case of inflaton. We can estimate the energy density in the AD field by $\rho_{\psi}$ $=\tilde{m}^{2} \psi_{0}^{2}\left(a_{\psi} / a\right)^{3}=\left(\tilde{m} \sqrt{\lambda} \psi_{0}^{2} / M_{p}\right)\left(a_{\lambda} / a\right)^{3}$. It can be easily verified that for larger $\psi_{0}, \Gamma_{\phi} / \Gamma_{\psi}>1$ for the sfermion decay rate $\Gamma_{\psi} \sim\left(\tilde{m}^{3} / \psi^{2}\right)$ [19]. However, in this case an important factor is that thermalization due to the decay of the inflaton field must happen after the full decay of the AD field.

Once the Universe becomes radiation dominated, the energy density of the relativistic decay products of the inflaton can be given by $\rho_{r \phi}=\left(m_{\phi}^{6} / M_{p}^{2}\right)\left(a_{d \phi} / a_{\lambda}\right)^{4}\left(a_{\lambda} / a\right)^{4}$ $=\left(\lambda^{4 / 3} M_{p}^{2 / 3} / m_{\phi}^{2}\right)\left(a_{\lambda} / a\right)^{4}$, and, the Hubble parameter is given by $H=\left(\lambda^{2 / 3} / m_{\phi} M_{p}^{2 / 3}\right)\left(a_{\lambda} / a\right)^{2}$. Now we must estimate when the AD field decays, following Refs. [18] and [19] we equate $H \sim \Gamma_{\psi} \equiv \tilde{m}^{3} / \psi^{2}$. This takes place when the scale factor is given by $a=a_{d \psi}=\left(\lambda^{7 / 6} \psi_{0}^{2} / \tilde{m}^{4} m_{\phi} M_{p}^{5 / 3}\right)^{1 / 5} a_{\lambda}$. It can be verified that $\rho_{r \phi}\left(a_{d \psi}\right)>\rho_{\psi}\left(a_{d \psi}\right)$. Now we have to make sure that the thermalization of the inflaton field happens after the decay of the the AD field. For that we need to estimate the thermalization rate of the inflaton field. Following the arguments given in Refs. [18] and [19] we get

$$
\begin{aligned}
& \Gamma_{T} \sim n_{\phi} \sigma \sim m_{\phi} \phi^{2}\left(a_{\phi} / a\right)^{3} \sim\left(\alpha^{2} / m_{\phi}^{2}\right)\left(a / a_{d \phi}\right)^{2} \\
& \sim \alpha^{2}\left(\lambda^{1 / 3} m_{\phi} / M_{p}^{4 / 3}\right)\left(a_{\lambda} / a\right),
\end{aligned}
$$

where $n_{\phi}$ is the number density of the relativistic particles, $\sigma$ is the cross section, and $\alpha$ is the fine structure constant. The thermalization of the Universe occurs when $\Gamma_{T} \sim H$ and $a_{T}$ $=\alpha^{-2}\left(\lambda^{1 / 3} M_{p}^{2 / 3} / m_{\phi}^{2}\right) a_{\lambda}$. At this point we can also check that $a_{d \psi}<a_{T}$ for $m_{\phi} \sim 10^{-5} M_{5}$, and $\alpha \sim 10^{-3 / 2}$. The condition is satisfied for any reasonable value of $\psi_{0}$ less than the fourdimensional Planck mass.

At $a_{T}$ we can compute the final baryon to entropy ratio given by Ref. [5]. We also have to compute the entropy, which is given by $s=\left[\rho_{r \phi}\left(a_{T}\right)\right]^{3 / 4} \approx\left(\alpha^{6} m_{\phi}^{9 / 2} / M_{p}^{3 / 2}\right)$ and finally the baryon to entropy ratio can be given by

$$
\frac{n_{B}}{s}=\frac{\epsilon \psi_{0}^{4} m_{\phi}^{3 / 2}}{M_{G}^{2} \sqrt{\lambda} M_{p}^{3 / 2}} \equiv \frac{\epsilon \psi_{0}^{4} m_{\phi}^{3 / 2}}{M_{G}^{2} M_{5}^{3} M_{p}^{1 / 2}} .
$$

It is noticeable that the baryon to entropy ratio does not depend on $\tilde{m}$. However, it does depend on the brane tension and the initial amplitude of the AD field oscillations. The last step in the above equation has been been expressed in terms of the five-dimensional Planck mass. For an example, we may take $M_{G} \sim 10^{15} \mathrm{GeV}, m_{\phi} \sim 10^{-5} M_{5}$, we get an estimation of the initial amplitude of oscillations in the AD field $\psi_{0}=\left(10^{37} / \epsilon\right)^{1 / 4}\left(M_{5} / \mathrm{GeV}\right)^{3 / 8} \mathrm{GeV}$, where we have taken the observed baryon to entropy ratio to be $n_{B} / s \sim 10^{-10}$. It is evident that the value of $\psi_{0}$ is more than $\phi_{\mathrm{COBE}} \approx 10^{2} M_{5}$. However, for smaller values of $M_{5}$ the amplitude could be comparable to $\phi_{\mathrm{COBE}}$. In that case, situation could be different. Here we have implicitly assumed that the AD field decays after the decay of the inflaton.

Here we summarize by saying that the brane-world cosmology differs quite a bit in their predictions from the standard cosmology. Here we have looked upon two issues, the gravitino abundance and the baryogenesis. Other interesting issues should also be taken into account and work in this direction is in progress.

The author is supported by INLAKS foundation. The author is thankful to Andrew Liddle for fruitful discussions.
[1] P. Horava and E. Witten, Nucl. Phys. B460, 506 (1996); B475, 94 (1996).

[2] A. Lukas, B. A. Ovrut, K. Stelle, and D. Waldram, Phys. Rev. D 60, 086001 (1999); J. E. Ellis, Z. Lalak, S. Pokorski, and W. Pokorski, Nucl. Phys. B540, 149 (1999).

[3] A. Lukas, B. A. Ovrut, and D. Waldram, Phys. Rev. D 61, 023506 (2000).

[4] P. Binetruy, C. Daffayet, and D. Langlois, Nucl. Phys. B565, 269 (2000); P. Binetruy, C. Daffayet, U. Ellwanger, and D. Langlois, Phys. Lett. B 477, 269 (2000).

[5] I. Affleck and M. Dine, Nucl. Phys. B249, 361 (1985).

[6] L. Randall and R. Sundrum, Phys. Rev. Lett. 83, 3370 (1999).

[7] R. Altendorfer, J. Bagger, and D. Nemeschansky, Phys. Rev. D 63, 125025 (2001); A. Falkowski, Z. Lalak, and S. Pokorski, Phys. Lett. B 491, 172 (2000).

[8] R. Kallosh and A. Linde, J. High Energy Phys. 02, 005 (2000); E. Bergshoeff, R. Kallosh, and A. Van Proeyen, ibid. 10, 033 (2000).

[9] M. J. Duff, J. T. Liu, and K. S. Stelle, hep-th/0007120.

[10] M. J. Duff, J. T. Liu, and W. A. Sabra, hep-th/0009212.
[11] J. M. Cline, C. Grojean, and G. Servant, Phys. Rev. Lett. 83, 4245 (1999).

[12] L. Randall and R. Sundrum, Phys. Rev. Lett. 83, 4690 (1999).

[13] R. Maartens, D. Wands, B. A. Bassett, and I. P. C. Heard, Phys. Rev. D 62, 041301 (2000); E. J. Copeland, A. R. Liddle, and J. E. Lidsey, astro-ph/0006421.

[14] E. W. Kolb and M. Turner, The Early Universe (AddisonWesley, New York, 1993); M. Kawasaki and T. Moroi, Prog. Theor. Phys. 93, 879 (1995); T. Moroi, Ph.D. thesis, hep-ph/9503210.

[15] J. Cline and S. Raby, Phys. Rev. D 43, 1781 (1991).

[16] A. L. Maroto and A. Mazumdar, Phys. Rev. Lett. 84, 1655 (2000); R. Kallosh, L. Kofman, A. Linde, and A. V. Proyen, Phys. Rev. D 61, 103503 (2000); G. F. Giudice, A. Riotto, and I. Tkachev, J. High Energy Phys. 08, 009 (1999).

[17] I. Oda, hep-th/0008134.

[18] J. Ellis, D. V. Nanopoulos, and K. A. Olive, Phys. Lett. B 184, 37 (1987)

[19] J. Ellis, K. Enqvist, D. V. Nanopoulos, and K. A. Olive, Phys. Lett. B 191, 343 (1987). 